\title{
LOCAL ISOTROPY AND ANISOTROPY IN THE SHEARED AND HEATED ATMOSPHERIC SURFACE LAYER
}

\author{
GABRIEL G. KATUL, ${ }^{1}$ MARC B. PARLANGE, ${ }^{2}$ JOHN D. ALBERTSON ${ }^{2}$ and \\ CHIA R. CHU ${ }^{3}$ \\ ${ }^{1}$ School of the Environment, Duke University, Durham, NC 27708-0328, U.S.A.; ${ }^{2}$ Hydrologic \\ Science, University of California at Davis, Davis, CA 95616, U.S.A.; ${ }^{3}$ Department of Civil \\ Engineering, National Central University, Chung-Li, Taiwan
}

(Received in final form 2 June, 1994)

\begin{abstract}
Longitudinal velocity and temperature measurements above a uniform dry lakebed were used to investigate sources of eddy-motion anisotropy within the inertial subrange. Rather than simply test the adequacy of locally isotropic relations, we investigated directly the sources of anisotropy. These sources, in a daytime desert-like climate, include: (1) direct interaction between the large-scale and small-scale eddy motion, and (2) thermal effects on the small-scale eddy motion. In order to explore these two anisotropy sources, we developed statistical measures that are sensitive to such interactions. It was found that the large-scale/small-scale interaction was significant in the inertial subrange up to 3 decades below the production scale, thus reducing the validity of the local isotropy assumption. The anisotropy generated by thermal effects was also significant and comparable in magnitude to the former anisotropy source. However, this thermal anisotropy was opposite in sign and tended to counteract the anisotropy generated by the large-scale/smallscale interaction. The thermal anisotropy was attributed to organized ramp-like patterns in the temperature measurements. The impact of this anisotropy cancellation on the dynamics of inertial subrange eddy motion was also considered. For that purpose, the Kolmogorov-Obukhov structure function equation, as derived from the Navier-Stokes equations for locally isotropic turbulence, was employed. The Kolmogorov-Obukhov structure function equation in conjunction with Obukhov's constant skewness closure hypothesis reproduced the measured second- and third-order structure functions. Obukhov's constant skewness closure scheme, which is also based on the local isotropy assumption, was verified and was found to be in good agreement with the measurements. The accepted 0.4 constant skewness value derived from grid turbulence experiments overestimated our measurements. A suggested 0.26 constant skewness value, which we derived from Kolmogorov's constant, was found to be adequate.
\end{abstract}

\section{Introduction}

The mechanisms by which a scalar is transported within the atmospheric surface layer (ASL) are of great importance in surface hydrology and land-atmosphere interaction studies. In particular, desert-like regions pose unique problems in describing these mechanisms since the daytime sensible heat flux $(H)$ from the ground, as well as the air temperature fluctuations, are large compared to more temperate environments. However, irrespective of the environment, all ASL flows are characterized by a long inertial subrange that extends over two or more decades of turbulent scales of motion. Within the inertial subrange, turbulent energy is neither produced nor dissipated but simply cascades down to smaller and smaller scales.

A useful feature of the inertial subrange is the isotropic nature of the eddy motion that permits simplifications to the statistical description of these scales of 
motion. Within the framework of Kolmogorov's (1941) theory (hereafter referred to as $\mathrm{K} 41$ ), it is argued that the many energy cascade steps eliminate all the turbulent production eddy-motion anisotropy and result in a locally isotropic turbulence structure. Therefore, the study of isotropy in the inertial subrange is important to many ASL transport phenomena, turbulence closure models, and energy-cascade models.

The wide inertial subrange in ASL flows is typically attributed to the high Reynolds number and associated wide scale separation between turbulent production (at $L_{u}$ ) and viscous dissipation (at $\eta$ ) (for many ASL flows $L_{u} / \eta \approx 10^{5}$ ). Here, $L_{u}$ is the integral length scale of the longitudinal velocity fluctuations, $\eta\left(=\left[\nu^{3} /\langle\epsilon\rangle\right]^{1 / 4}\right)$ is the Kolmogorov microscale, $\nu\left(=1.5 \times 10^{-5} \mathrm{~m}^{2} \mathrm{~s}^{-1}\right)$ is the kinematic viscosity of air, $\epsilon\left(=\nu\left[\partial u_{i} / \partial x_{j}+\partial u_{j} / \partial x_{i}\right]^{2}\right)$ is the turbulent kinetic energy dissipation rate, $u_{i}$ are the turbulent velocity fluctuation components $\left(u_{1}=u_{2} u_{2}=v\right.$, and $\left.u_{3}=w\right), x_{1}(=x), x_{2}(=y)$, and $x_{3}(=z)$ and $x_{3}(=z)$ are the longitudinal, lateral, and vertical directions, respectively (in this study, both meteorological and tensor notations are used), and $\langle$.$\rangle is the time averaging oper-$ ator (assumed to be identical to the ensemble averaging operator). The wide separation between $L_{u}$ and $\eta$ ensures that the transfer of turbulent kinetic energy $\left(T_{E}\right)$, from production to dissipation, cascades over many intermediate scales. It is expected that all the anisotropy in the eddy motion associated with the turbulent production (mechanical or thermal) should diminish during this energy cascade.

In the past two decades, various laboratory studies (e.g,. Mestayer, 1982; Sreenivasan, 1991) have suggested that a long inertial subrange, in the sense of $\mathrm{K} 41$, may not necessarily result in isotropic eddy motion for inertial subrange scales (referred to as small scales in this paper). It is now recognized that the breakdown of local isotropy is, in part, attributed to direct interaction between the energy-containing scales and the small scales (see e.g., Mahrt, 1989; Sreenivasan et al., 1979). Furthermore, laboratory experiments (e.g., Antonia and Van Atta, 1975) as well as field studies (e.g., Antonia and Chambers, 1980) have demonstrated that small-scale anisotropy may also occur due to thermal influences. Yet, despite the potential sources of anisotropy, results regarding the validity of local isotropy were obtained from the Kansas experiments (Kaimal et al., 1972) and many other ASL experiments (see Monin and Yaglom, 1975, Ch. 8). More recently, direct numerical simulations of open channel flow by Kim and Antonia (1993) indicate that local isotropy is achieved even at moderate Reynolds number with no apparent inertial subrange. Table I summarizes results from several laboratory and ASL experiments related to the existence of local isotropy in the inertial subrange. Many more experiments have been reported in the literature but will not be reviewed here. Also, it should be noted that the methods used to evaluate local isotropy varied across different studies (see e.g., Van Atta, 1991 for consequences). 
TABLE I

Summary of some local isotropy experiments

\begin{tabular}{lll}
\hline Reference & Experiment/Conditions & Inertial subrange \\
\hline Monin and Yaglom (1975) & ASL flows (land and ocean) & Isotropic \\
Kaimal $e$ t al. (1972) & Stable and unstable ASL flows & Isotropic \\
Mestayer (1982) & Heated boundary layer & No isotropy \\
Sreenivasan et al. (1979) & Heated axisymmetric jet & No isotropy \\
Van Atta (1991) & Stably-stratified wind tunnel boundary & No isotropy \\
& layer & \\
Van Atta (1991) & Neutral wind tunnel boundary layer & Isotropic \\
Antonia and Raupach (1993) & Neutral wind tunnel boundary layer & No isotropy \\
\hline
\end{tabular}

It is these conflicting results that have motivated the present study. In particular, we consider the various mechanisms responsible for anisotropy in the inertial subrange. If the interaction between large and small scales is important, and/or if the influence of temperature on the inertial subrange isotropy is significant, then studying the inertial subrange of a desert-like environment is ideal for this investigation. Such environments possess two desirable features: (1) the Reynolds number is very large and ensures a wide inertial subrange, and (2) the surface heating, sensible heat flux, and temperature variance are all very large.

An experiment was carried out above a uniform dry lakebed (Owen's Lake) in Owen's Valley, California. Simultaneous longitudinal velocity and temperature measurements were carried out above the lakebed surface to investigate the statistical structure of the inertial subrange of the longitudinal velocity. The specific objectives of our investigation are to study (1) the interaction between the largescale and small-scale eddy motions along the longitudinal direction, (2) the influence of temperature on the inertial subrange of the longitudinal velocity, and (3) the influence of anisotropy on equations that utilize local isotropy simplifications to the dynamics of inertial subrange eddy motion. The Kolmogorov-Obukhov structure function equation is a good surrogate for investigating (3) since it is rigorously derived from the Navier-Stokes (NS) equations following local isotropy simplifications. Finally, we discuss the performance of some closure assumptions of this equation.

\section{Theory}

The dynamic equation relating the second- and third-order structure functions, as derived from NS equations for locally isotropic turbulence, is given by

$$
D^{3}(r)-6 \nu \frac{\mathrm{d}}{\mathrm{d} r} D^{2}(r)=-\frac{4}{5}\langle\epsilon\rangle r
$$


where $D^{n}(r)=\left\langle[u(x+r)-u(x)]^{n}\right\rangle$, and $r$ is the separation distance along the longitudinal direction (see Monin and Yaglom, 1975: pp. 401-403). In deriving (1), it was assumed that the statistical state of the small-scale eddies (i.e., scales much smaller than $L_{u}$ ) are independent of the macro-structural flow properties. Also, as discussed in Monin and Yaglom (1975: p. 402), it was assumed in (1) that the temperature and velocity differences ( $\Delta T$ and $\Delta u$ ), for $r$ much less than $L_{u}$, are independent. That is, at the small scales, temperature is a passive scalar and does not interact with the velocity field. Throughout this study, we shall assume that at any time instant, the longitudinal velocity $(U)$ and air temperature $\left(T_{a}\right)$ can be decomposed, without ambiguity, into time averages $(\langle U\rangle,\langle T\rangle)$ and fluctuations about these averages $(u, T)$. Thus, at time $t, U(t)=\langle U\rangle+u(t)$ and $T_{a}(t)=\langle T\rangle+T(t)$. An important asymptotic result that follows from (1) is the behavior of $D^{3}(r)$ in the limit when $r \gg \eta$ and $\nu$ is very small. This is given by

$$
D^{3}(r) \simeq-\frac{4}{5}\langle\epsilon\rangle r
$$

which agrees well with K41 scaling $\left[D^{n}(r) \propto(\langle\epsilon\rangle r)^{n / 3}\right]$ for $n=3$. In general, (1) is not closed since it involves two unknown functions: $D^{2}(r)$ and $D^{3}(r)$. To close (1), one must introduce a hypothesis to express one unknown as a function of the other unknown. Obukhov (1949) proposed a simple closure hypothesis known as the hypothesis of constant skewness, and thus, established a relation between $D^{2}(r)$ and $D^{3}(r)$. Obukhov's constant skewness hypothesis assumes that the skewness

$$
S(r)=\frac{D^{3}(r)}{\left[D^{2}(r)\right]^{3 / 2}}
$$

is a negative constant independent of $r$. The value of this constant $(|S|)$ was determined experimentally to be $0.2-0.5$ (see footnote Landau and Lifshitz, 1986: pp. 128; Katul et al., 1994a; Townsend, 1975: p. 98). With this closure assumption, (1) simplifies to

$$
|S|\left[D^{2}(r)\right]^{3 / 2}+6 \nu \frac{\mathrm{d}}{\mathrm{d} r} D^{2}(r)=\frac{4}{5}\langle\epsilon\rangle r
$$

which is only a function of $D^{2}(r)$. Hence, if $\langle\epsilon\rangle$ is known, (4) can be used to solve for $D^{2}(r)$, and then (3) can be used to compute $D^{3}(r)$. Again, we remark that $|S|$ cannot be a constant if local isotropy is violated (see Landau and Lifshitz, 1986: p. 128). Notice here the key difference between (1) and K41. In K41, the statistical properties of locally isotropic turbulence were investigated by dimensional analysis which do not require the explicit use of the fluid dynamics equations, while (1) was derived from NS equations by invoking the local isotropy assumption to simplify various dynamical terms and determine the universal constants (see Monin and Yaglom, 1975: pp. 401-403 for derivation). In the results and discussion section that follows, we focus on the performance of (3) and (4) as well as the onset of local isotropy. But first, we present the experimental setup. 


\section{Experimental Setup}

The measurements discussed in the present study were carried out on June 27, 28, and 29 (1993) over a uniform sandy dry lakebed (Owen's lake) in Owen's valley, California. The lakebed (elevation $=1100 \mathrm{~m}$ ) is contained within a larger basin bounded by the Sierra Nevada range and the White and Inyo Mountains. The surface is a uniform heaved sandy soil extending some $11 \mathrm{~km}$ in the NorthSouth direction and $4 \mathrm{~km}$ in the East-West direction (see Katul, 1994; Katul et al., 1994b). The predominant wind direction was along the North-South direction.

The three velocity components were measured using a triaxial ultrasonic anemometer (Gill Instruments/1012R2). Sonic anemometers achieve their frequency response by sensing the effect of wind on the transit times of sound pulses traveling in opposite directions across a known instrument path distance $d_{s i}(=0.149 \mathrm{~m}$ in this study). The sonic anemometer is well suited for field experiments since it is relatively free of calibration nonlinearities and atmospheric contamination drifts. As discussed by Friehe (1986), Suomi and Businger (1959), and Wyngaard (1981), the primary disadvantage of sonic anemometers is the wavenumber distortion due to averaging along the finite sonic path $d_{s l}$. This distortion is restricted to wavenumbers in excess of $2 \pi / d_{s l}\left(=42.2 \mathrm{~m}^{-1}\right.$ in this study) (see Wyngaard, 1981). The sampling frequency $\left(f_{s}\right)$ and the sampling period $\left(T_{p}\right)$ were $56 \mathrm{~Hz}$ and $15 \mathrm{~min}$, respectively, resulting in 50,400 measurements per velocity component. The $56 \mathrm{~Hz}$ sampling frequency is the maximum achievable frequency by the Gill sonic anemometer. The short sampling period was necessary to insure steady state mean meteorological conditions. Taylor's (1938) hypothesis was used to convert time increments to space increments $(\mathrm{d} r=-\langle U\rangle$ $\mathrm{d} t$ ). We note that some distortion occurs for $r<d_{s l}$ and we limit our analysis to $r>d_{s l}$ but show the full range of measurements. From this experiment, twenty 15-min runs were collected under unstable and stable atmospheric stability conditions (see Katul, 1994 for further details). Here, we focus on three unstable atmospheric stability runs that exhibited: (1) at least 1.5 decades of inertial subrange as identified by the third-order structure function, (2) a turbulent intensity not exceeding 0.4 to insure the applicability of Taylor's hypothesis (see Stull, 1988: p. 6), (3) a temperature standard deviation in excess of $0.1^{\circ} \mathrm{C}$ to insure adequate thermal agitation, and (4) a clear and identifiable time average to permit decomposition into a mean and a fluctuating part without ambiguity.

The temperature was determined from the speed of sound $c_{s}$ using

$$
c_{s}^{2}=\alpha R_{d} T_{a},
$$

where $R_{d}\left(=287.04 \mathrm{~J} \mathrm{Kg}^{-1} \mathrm{~K}^{-1}\right)$ is the gas constant of dry air, and $\alpha(=1.4)$ is the ratio of the molar specific heat capacities of air at constant pressure to that at constant volume. The variations of $U$ and $T_{a}$ for all three runs are shown in Figures $1 \mathrm{a}, 1 \mathrm{~b}$, and $1 \mathrm{c}$, respectively. Notice that a linear trend is apparent in the temperature time series of Figure 1c, and therefore, the temperature measurements 

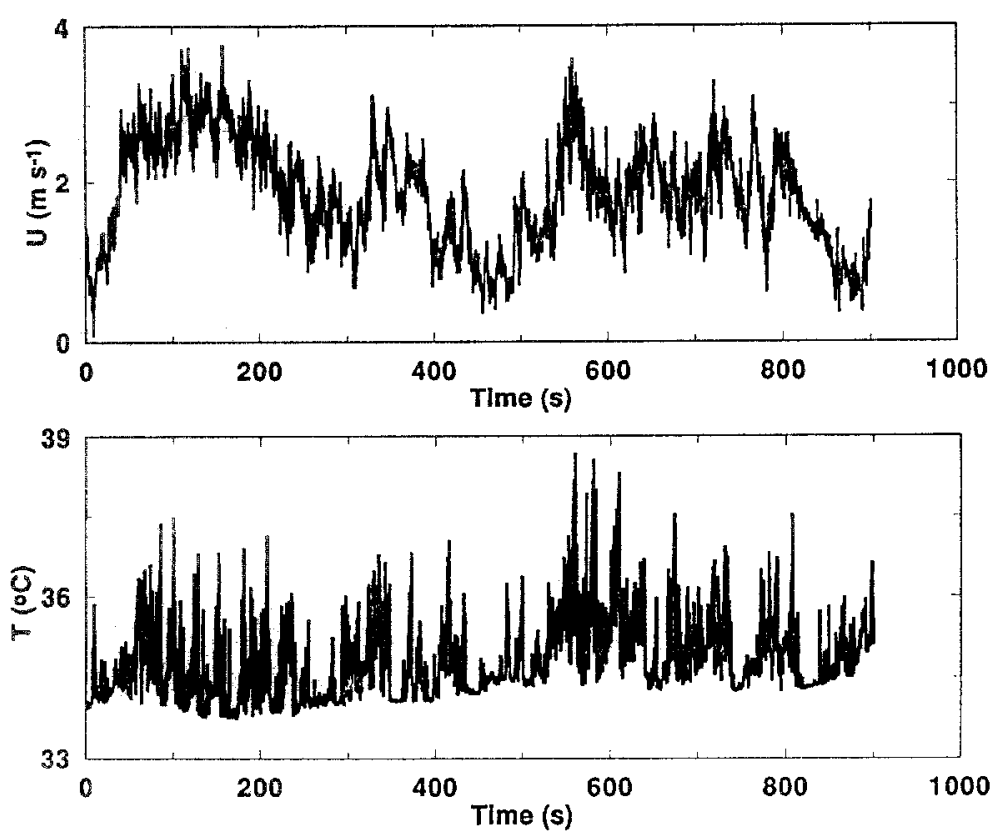

Fig. 1a. Time variation of the sonic anemometer measured longitudinal velocity and temperature for Run $\sharp 1\left(z / L_{\mathrm{MO}}=-1.06\right)$. The sampling frequency is $56 \mathrm{~Hz}$ and the sampling period is 15 $\min (N=50,400)$.

for Run $\sharp 3$ were de-trended. The mean meteorological and turbulence conditions are presented in Table II. In Figure $1 \mathrm{~b}$ (Run $\sharp 2$ ), velocities up to $14 \mathrm{~m} \mathrm{~s}^{-1}$ (peakto-mean ratio $=2.3$ ) and air temperatures up to $47.5^{\circ} \mathrm{C}$ (peak-to-mean ratio $=$ 1.15) were recorded.

To check how well the sonic anemometer captures the temperature fluctuations, a comparison between temperature determined from the sonic anemometer and temperature measured by a fine wire thermocouple (TC diameter $=0.0127$ $\mathrm{mm}$ and placed $60 \mathrm{~cm}$ east of the sonic anemometer) is shown in Figure 2a. The temperature measurements from the sonic anemometer are de-meaned. The TC measurements are shifted by $10^{\circ} \mathrm{C}$ to permit comparison. The sampling frequency in Figure $2 \mathrm{a}$ was $10 \mathrm{~Hz}$ and the height above the ground surface was $2.5 \mathrm{~m}$. Notice that all major events are captured well by both instruments. The power spectra for both signals are compared in Figure $2 \mathrm{~b}$. The TC spectrum is shifted by one decade along the ordinate to permit comparison at the high frequency end. At the low frequency end, both instruments are in excellent agreement. However, at the high frequency end, the TC spectrum appears to "level off" due to limited resolution (indicative of white noise). The sonic-anemometer temperature spectrum did not level off. This comparison demonstrates that the sonic anemometer temperature measurements are adequate for our study (see also Katul, 1994). 

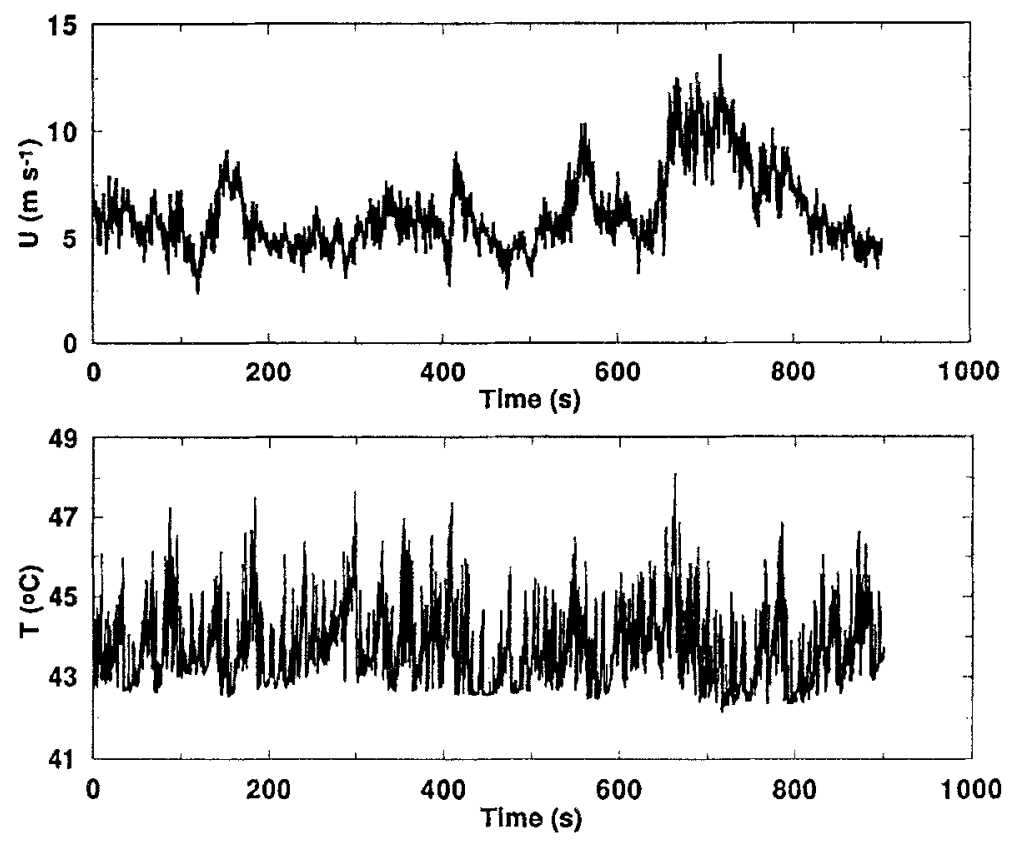

Fig. 1b. Same as Figure 1a but for Run $\sharp 2\left(z / L_{\mathrm{MO}}=-1.40\right)$.
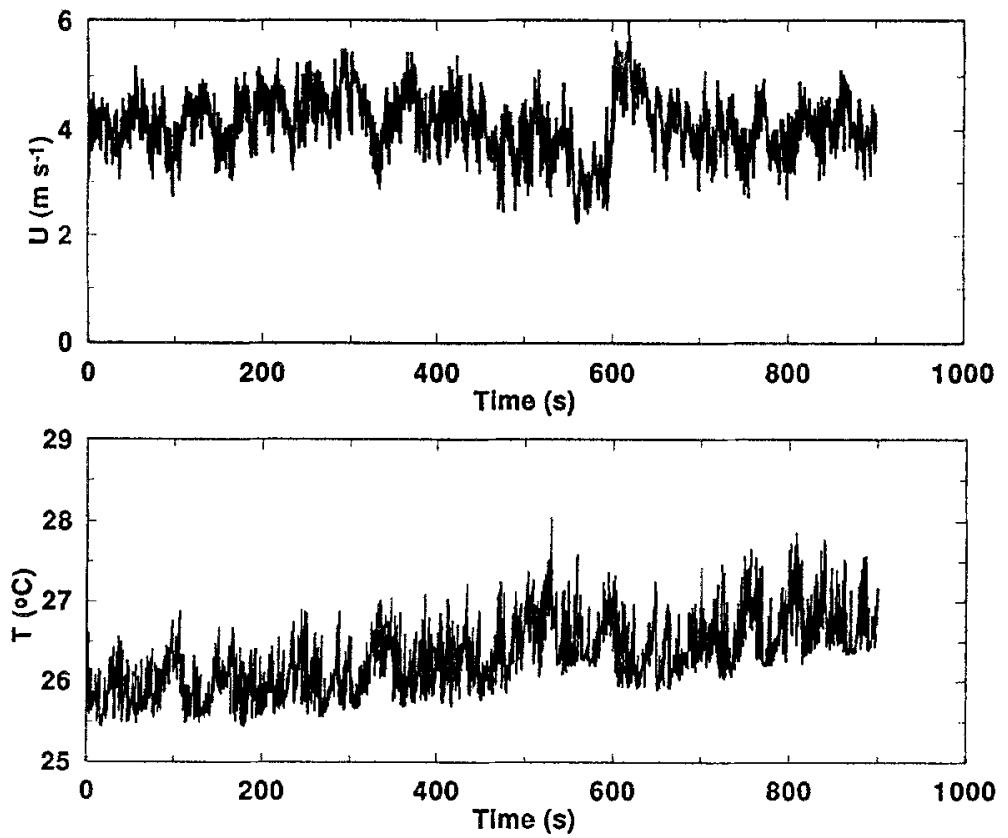

Fig. 1c. Same as Figure 1a but for Run $\sharp 3\left(z / L_{\mathrm{MO}}=-0.17\right)$. 
TABLE II

Summary of measured mean meteorological and turbulence conditions

\section{Run description}

Run

Day of year (1993)

Time (PDST)

$\begin{array}{lll}1 & 2 & 3 \\ 178 & 179 & 180 \\ 08: 53 & 17: 10 & 07: 42\end{array}$

Mean meteorological conditions

Net radiation $\left(R_{n}\right), \mathrm{W} \mathrm{m}^{-2}$

$227 \quad 245 \quad 94$

Sensible heat flux $(H), \mathrm{W} \mathrm{m}^{-2}$

Long. velocity $\left\langle U_{1}\right\rangle, \mathrm{m} \mathrm{s}^{-1}$

1.86

6.16

4.09

Air temperature $\left(T_{s}\right),{ }^{\circ} \mathrm{C}$

34.8

43.7

Turbulence statistics

Root-mean square velocity $\left(\sigma_{u}\right), \mathrm{m} \mathrm{s}^{-1}$

$\begin{array}{lll}0.665 & 1.79 & 0.533\end{array}$

Root-mean square velocity $\left(\sigma_{v}\right), \mathrm{m} \mathrm{s}^{-1}$

Root-mean square velocity $\left(\sigma_{w}\right), \mathrm{m} \mathrm{s}^{-1}$

Root-mean square temperature $\left(\sigma_{T}\right),{ }^{\circ} \mathrm{C}$

0.51

0.72

0.18

Friction velocity $\left(u_{*}\right), \mathrm{m} \mathrm{s}^{-1}$

0.15

0.17

0.18

Buoyant production rate $\left(P_{b} \times 10^{3}\right), \mathrm{m}^{2} \mathrm{~s}^{-3}$

3.03

Mechanical production rate $\left(P_{s} \times 10^{3}\right), \mathrm{m}^{2} \mathrm{~s}^{-3}$

2.85

Total production rate $\left(P \times 10^{3}\right), \mathrm{m}^{3} \mathrm{~s}^{-3}$

Mean dissipation rate $\left(\langle\epsilon\rangle \times 10^{3}\right), \mathrm{m}^{2} \mathrm{~s}^{-3}$

Method II: Local isotropy

$2.5 \quad 11.6$

Method III: Power spectrum

$3.1 \quad 14.3$

Turbulent intensity $\left(I_{u}\right)$

0.36

0.29

0.13

Length scales

Height $(z), \mathrm{m}$

Obukhov length $\left(L_{\mathrm{MO}}\right), \mathrm{m}$

Integral length scale $\left(L_{u}\right), \mathrm{m}$

$\begin{array}{lll}3.0 & 3.5 & 2.25\end{array}$

$\begin{array}{lll}-2.8 & -2.5 & -13.2\end{array}$

Integral length scale $\left(L_{T}\right), \mathrm{m}$

$61 \quad 272$

43

$35 \quad 33$

10

Taylor microscale $(\lambda), \mathrm{m}$

$0.26 \quad 0.25$

0.14

Kolmogorov dissipation scale $(\eta), \mathrm{mm}$

Flow properties

Reynolds number $\left(\operatorname{Re}_{\lambda}\right)$

$\begin{array}{lll}11,398 & 29,702 & 4953\end{array}$

Scale separation $\left(L_{u} / \eta\right) \times 10^{5}$

$0.50 \quad 3.70$

0.43

Stability parameter $\left(z / L_{\mathrm{MO}}\right)$

$\begin{array}{ll}-1.06 & -1.40\end{array}$

Inertial subrange $(\mathrm{m})$

$0.15-25 \quad 0.15-100$

$0.15-10$ 


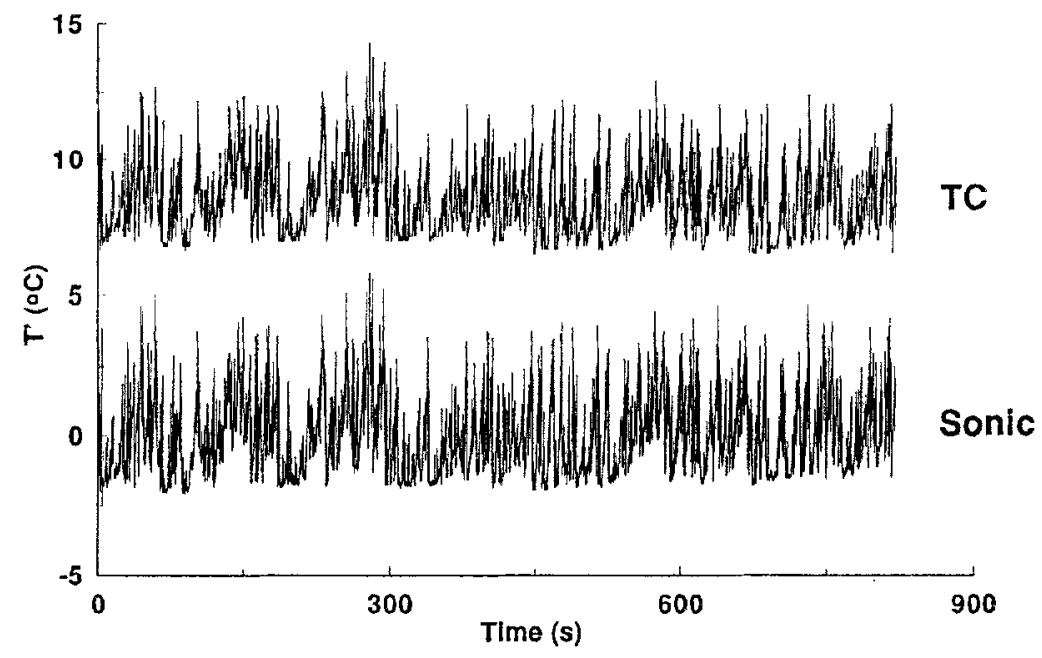

Fig. 2a. Comparison between thermocouple (TC) and sonic anemometer measured temperature. The TC is $60 \mathrm{~cm}$ away but at the same height.

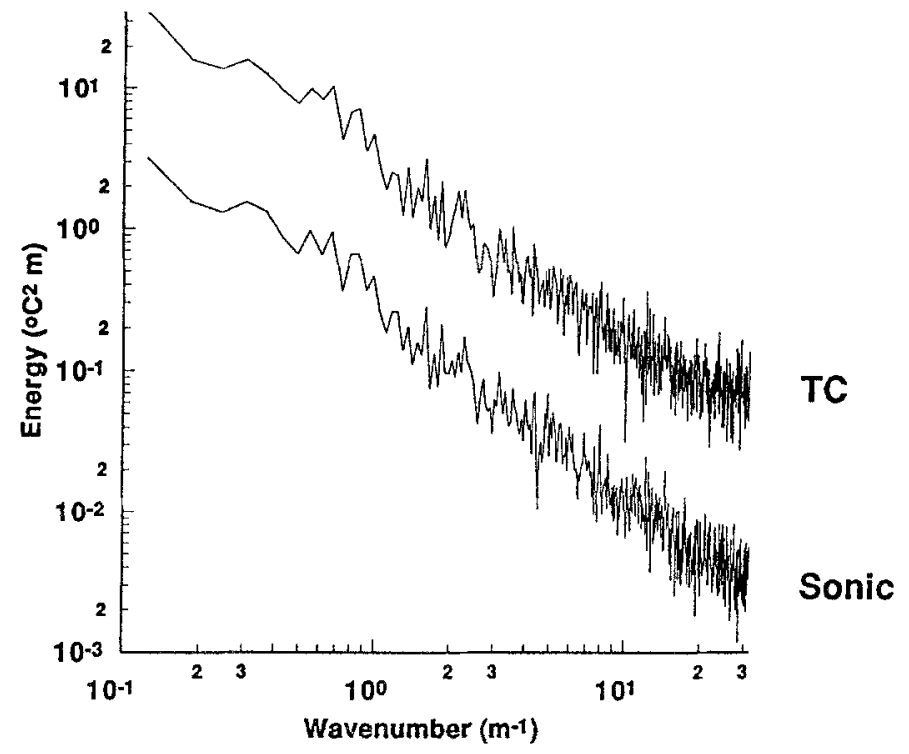

Fig. 2b. Comparison between thermocouple (TC) and sonic anemometer power spectra. The two spectra were computed from the time series of Figure 2a but separated by one decade to permit comparison at large wavenumbers.

Figures $1 \mathrm{a}, 1 \mathrm{~b}$, and $1 \mathrm{c}$ clearly indicate ramp-like structures in the temperature time series for all three stability cases (see Table II). As noted by Sreenivasan et al. (1979), these structures are commonly observed in shear flow experiments and are absent when the mean shear is zero. Therefore, these structures are typ- 


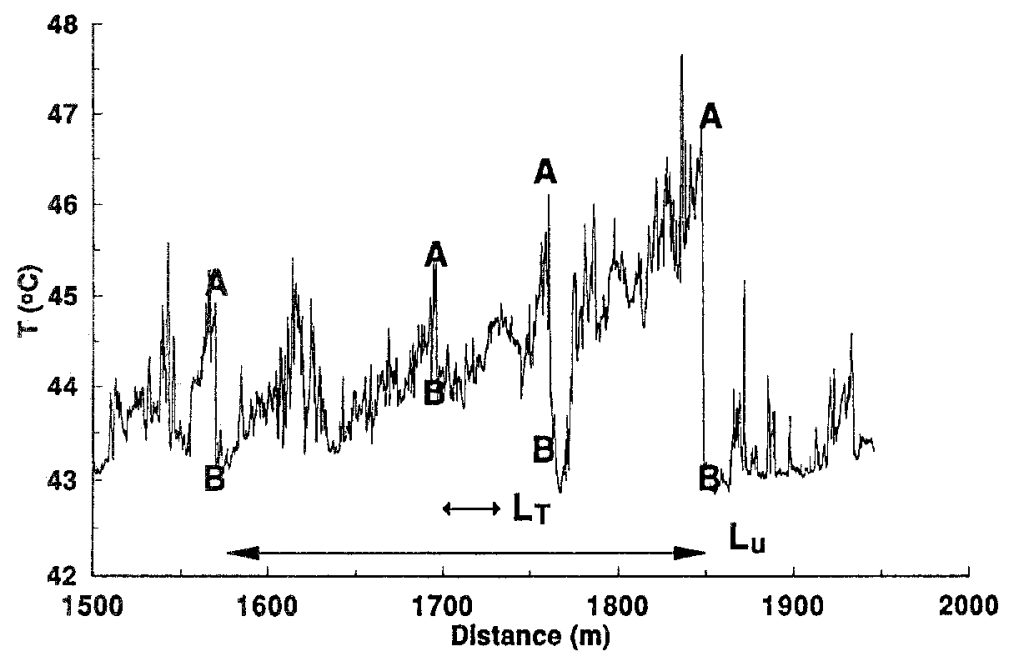

Fig. 3. A $500 \mathrm{~m}$ section of the temperature record of Run $\sharp 2$ to illustrate the ramp-like pattern and the sharp edges $(\mathrm{AB})$. For comparison purposes, the magnitude of the velocity and temperature integral length scales ( $L_{u}$ and $\left.L_{T}\right)$ are also shown. Taylor's hypothesis was used to convert time increments to space increments.

ically present in a desert-like environment during daytime conditions. To further illustrate the characteristics of these ramp-like patterns in the temperature measurements, we show in Figure 3 a small section of the record from Run $\sharp 2(\sim 500$ m) where Taylor's hypothesis was used to convert time to distance. Notice in Figure 3 that the size of the large ramp structure is about $100 \mathrm{~m}$ ( thickness of ASL) which is in agreement with many other ASL experiments (see Stull, 1988: pp. 442-446 for a review). Our interest in these structures stems from the conclusions of Sreenivasan et al. (1979), who demonstrated that the sharp edges of these ramps have important consequences on the isotropy of inertial subrange thermal motion.

The characteristic turbulence length scales are summarized in Table II, where $L_{u}$, the temperature integral length scale $\left(L_{T}\right)$, the Taylor microscale $(\lambda)$, and $\eta$ were estimated from

$$
\begin{aligned}
& L_{T}=\frac{\langle U\rangle}{\left\langle T^{2}\right\rangle} \int_{0}^{\infty}\langle T(t+\tau) T(t)>\mathrm{d} \tau, \\
& L_{u}=\frac{\langle U\rangle}{\left\langle u^{2}\right\rangle} \int_{0}^{\infty}\langle u(t+\tau) u(t)>\mathrm{d} \tau, \\
& \lambda=\frac{\sigma_{u}\langle U\rangle}{\left\langle\left(\frac{\partial u}{\partial t}\right)^{2}\right\rangle^{1 / 2}}
\end{aligned}
$$




$$
\eta=\left(\frac{\nu^{2}\langle U\rangle^{2}}{15\left\langle\left(\frac{\partial_{3 i}}{\partial t}\right)^{2}\right\rangle}\right)^{1 / 4}
$$

(see Tennekes and Lumley, 1972: pp. 66-67). For determining $L_{u}$ and $L_{T}$, the integration was carried out up to the first zero crossing as discussed by Sirivat and Warhaft (1983). Notice in Table II that both $L_{u}$ and $L_{T}$ are smaller for the near neutral run (Run $¥ 3$ ) when compared to the unstable runs (Runs 1 and 2). Also notice that $L_{T}$ (for Run $\ddagger 2$ ) is smaller than the typical ramp-like thermal structure of Figure 3.

The local isotropy assumption and the insufficient sampling resolution greatly influence the accuracy of $\lambda(=0.14 \mathrm{~m}-0.26 \mathrm{~m})$. However, the magnitude obtained in this study is comparable to other reported values. For example, Yamada and Ohkitani (1991) found that $\lambda=0.31 \mathrm{~m}$ at $15 \mathrm{~m}$, and Bradley et al. (1981) found that $\lambda$ varied between 0.06 and $0.14 \mathrm{~m}$ for unstable atmospheric conditions. The mean dissipation rates in Table II were estimated using three methods as discussed in Appendix 1.

The maximum turbulent intensity $\left(I_{u}\right)$ is about 0.36 (see Table II), which is not very large (see Stull, 1988: p. 6). Hence, Taylor's (1938) hypothesis does not cause significant distortion, at least for inertial subrange scales (e.g., Lumley, 1965 ) for all three runs. Other supporting mean meteorological measurements such as net radiation $\left(R_{n}\right), H$, and mean air temperature $\langle T\rangle$ are also presented in Table II for all three runs. Notice from Table II that the stability parameter $\left(z / L_{\mathrm{MO}}\right)$ varied by one order of magnitude. Finally, this experiment differs from many laboratory investigations because: (1) turbulence is produced in the vertical (buoyant production) and horizontal (shear production) directions for all three runs, (2) the Taylor microscale Reynolds number $\left(\operatorname{Re}_{\lambda}=\lambda \sigma_{u} / \nu \sim 10^{5}\right)$ is larger than in most laboratory flows, and (3) the scale separation between turbulent production and dissipation is also very large $\left(\sim 10^{6}\right)$ resulting in a wide inertial subrange (1.5-3 decades).

\section{Results and Disussion}

The results and discussion are presented in two parts. The first part evaluates the performance of (4) and the validity of the constant skewness hypothesis. This evaluation is important since any anisotropy within the inertial subrange adversely affects the performance of (3) and (4). The second part investigates some of the mechanisms potentially responsible for anisotropy in the inertial subrange. Namely, assumptions involving the interaction between the large scale turbulent motion and the small scales, and the interaction between the temperature and small scale velocity fluctuations. Our analysis diverges from previous investiga- 


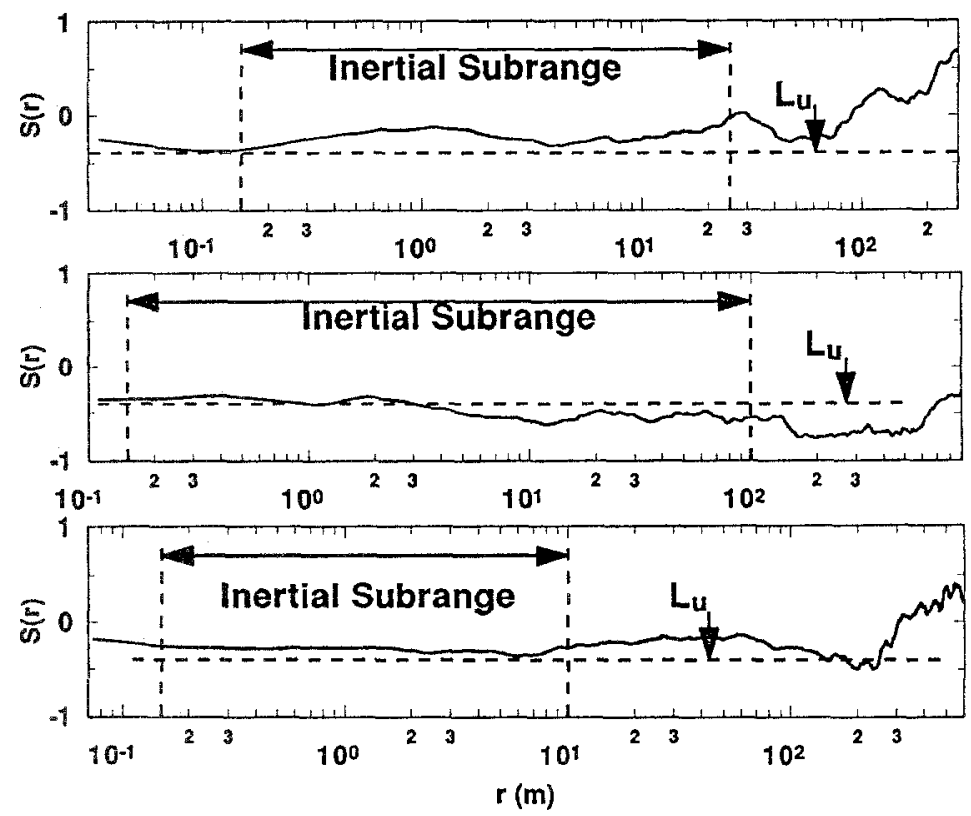

Fig. 4a. Validation of Obukhov's constant skewness hypothesis. The line $|S|=0.4$, suggested in Landau and Lifshitz (1986), is also shown (dotted line). The upper, middle, and lower figures are for Runs 1,2 , and 3, respectively.

tions since the interest is in the source of anisotropy rather than the consequence of anisotropy on well established isotropic relations.

\subsection{ASSESSMENT OF THE CONSTANT SKEWNESS HYPOTHESIS}

As noted earlier, the constant skewness hypothesis cannot be valid for anisotropic eddy motion. The validity of the constant skewness hypothesis of (3) was directly checked by determining $S(r)\left(=D^{3}(r)\left[D^{2}(r)\right]^{-3 / 2}\right)$ for all $r$ within the inertial subrange. The identification of the inertial subrange is discussed in Appendix 2. These results are summarized in Figure 4a, which displays the variation of $S(r)$ with $r$. From Figure $4 \mathrm{a}$, the accepted $|S|=0.4$ (dotted line) slightly overestimates our measurements for Runs 1 and 3. We should note here that the accepted 0.4 value was obtained from grid turbulence measurements (see Lesieur, 1987: p. 93) rather than ASL measurement. Direct numerical simulations at moderate Reynolds number by Kerr (1990) yielded an $|S|=0.5$, while Townsend (1976) suggested a range of $|S|$ values between $0.22-0.30$, which agrees better with the data in Figure 4a. Monin and Yaglom (1975: pp. 471-472) report a range of $|S|$ from many ASL experiments between 0.2-0.45. For that purpose, we decided to derive a relation between $|S|$ and some well established inertial subrange constant. 


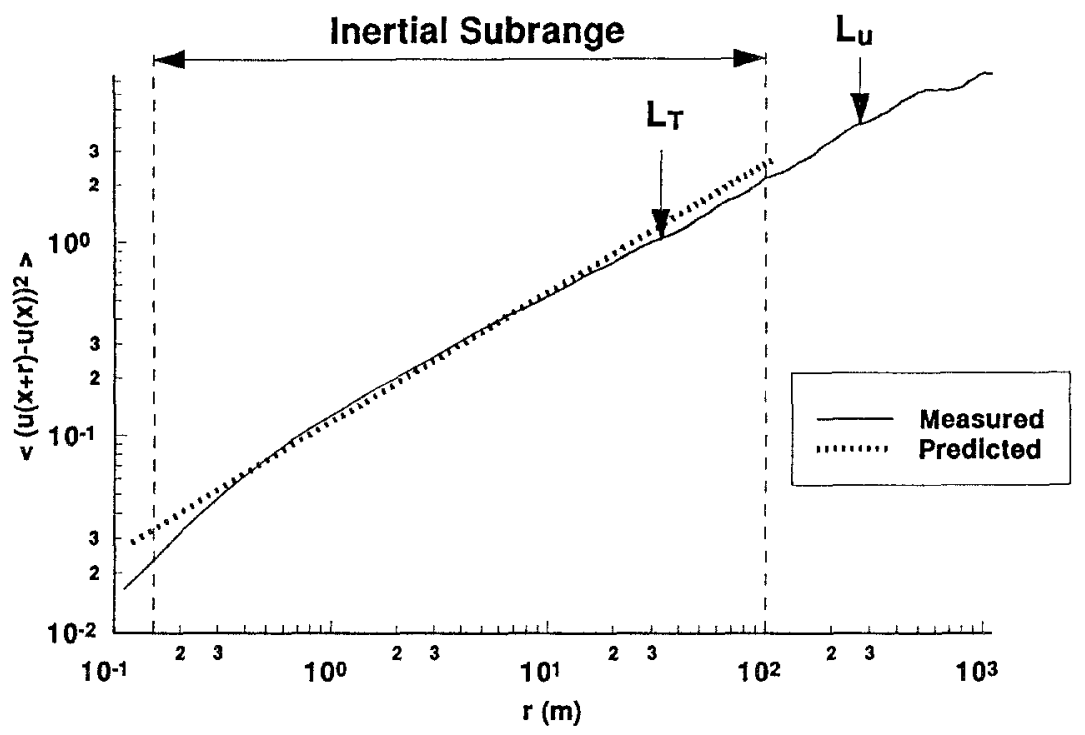

Fig. 4b. Comparison between predicted (dotted) and measured (solid) second-order structure function $\left[D^{2}(r)\right]$ for Run $\sharp 2$. The integral length scales of temperature and longitudinal velocity are also marked.

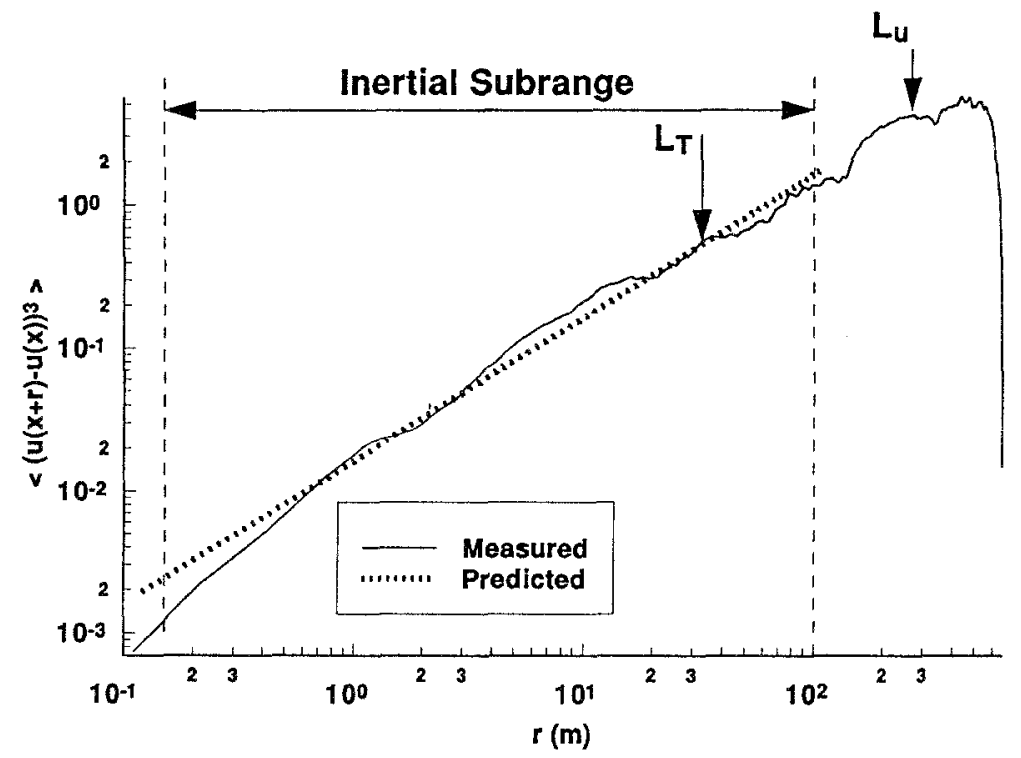

Fig. $4 \mathrm{c}$. Same as Figure $4 \mathrm{~b}$ but for the third-order structure function $\left[D^{3}(r)\right]$ and Run $\sharp 2$.

In Appendix 3, a relation between $|S|$ and the Kolmogorov constant $\left(C_{1}=\right.$ $0.55)$ is derived, resulting in an $|S|=0.25$. This value is also in agreement with the range reported by Townsend (1976) and Van Atta and Chen (1970) for the oceanic ASL. The measured sign of $S$ is also negative within the inertial subrange 
and is consistent with the fact that net energy transfer $T_{E}$ is being directed from the large scales to the small scales. The necessity of this becomes evident if we rearrange (2) as

$$
T_{E}=\langle\epsilon\rangle=-\frac{5}{4} \frac{D^{3}(r)}{r} .
$$

Since the net dissipation is always positive in turbulent flows, then from (7), $D^{3}(r)$ must be negative, and following from (3), $S$ must also be negative as $D^{2}(r)$ is positive. The good agreement between predictions and measurements of $|S|$ noted in Figure 4a indicates that: (1) the constant skewness hypothesis may be a valid working approximation for ASL flows, and (2) the 0.25 value for $|S|$ is reasonable. Having reinforced the validity of the closure assumption in (3), we solve (4) for $D^{2}(r)$ using Run $\sharp 2$ as an illustration:

(1) $D^{2}(0)=0$.

(2) $|S|=0.25$.

(3) $\langle\epsilon\rangle=0.012 \mathrm{~m}^{2} \mathrm{~s}^{-3}$ (see Table II and Appendix 1).

The integration of (4) was carried out via a fourth-order Runge-Kutta method with a step size of $\eta / 10$. A comparison between $D^{2}(r)$ predicted from the integration of (4) and $D^{2}(r)$ measured by the triaxial sonic anemometer is shown in Figures $4 \mathrm{~b}$ (for Run $\sharp 2$ ). Using the $D^{2}(r)$ predicted from (4) and the constant skewness hypothesis (with $|S|=0.25$ ), the magnitude of $D^{3}(r)$ was calculated and compared with the sonic anemometer measurements in Figure 4c. Good agreement between measured and predicted $D^{2}(r)$ and $D^{3}(r)$ is apparent in Figures $4 \mathrm{~b}$ and $4 \mathrm{c}$ except for $r<0.5 \mathrm{~m}$ (for Run $\sharp 2$ ). Similar results were obtained for Runs 1 and 3 (not shown here). Since (1) and the constant skewness hypothesis both rely on the local isotropy assumption, one may be inclined to conclude from these results that local isotropy is a valid assumption in the ASL. We consider the anisotropy next.

\subsection{Departures from local isotropy}

In this section, we focus on possible departures from isotropy at the small scales due to: (1) the large-scale/small-scale interaction, and (2) thermal influences on the longitudinal velocity fluctuations. The local isotropy assumption in (1) requires the absence of any interaction between the large and small scales of the velocity field, and (2) the absence of any interaction between the temperature and velocity field. If such interaction exists, then the anisotropy of the largescale eddy motion (or any thermal inhomogeneity) induces anisotropy on the small-scale eddy motion directly.

To study these interactions, we construct specific statistical quantities based on the following argument: at any position $x$, the velocity of the small-scale eddy motion (eddies of size $r$ ), within the inertial subrange, is characterized by the velocity difference $[\Delta u=u(x+r)-u(x)]$ over separation $r$. A comprehensive study regarding the validity of this characteristic velocity scale can be found in 
Praskovsky et al. (1993), Frisch et al. (1978), and Monin and Yaglom (Chap. 8). We assume that this argument can be extended to temperature as well.

\subsubsection{Large-Scale and Small-Scale Longitudinal Velocity Interaction}

A measure of the net interaction between the large-scale and the small-scale motions (characterized by $\Delta u$ ) is given by the correlation coefficient $\rho_{u, \Delta u}$

$$
\rho_{u, \Delta u}(r)=\frac{\langle(u(x)-\langle u(x)\rangle)(\Delta u(x, r)-\langle\Delta u(x, r)\rangle)\rangle}{\sigma_{u} \sigma_{\Delta u}}
$$

(see Praskovsky, 1992; and Praskovsky et al., 1993), where $\sigma_{x}$ is the standard deviation of the flow variable $x$ and is given by

$$
\sigma_{x}=\left\langle(x-\langle x\rangle)^{2}\right\rangle^{1 / 2} .
$$

The absence of net interaction between large- and small-scale eddy motions requires $\rho_{u, \Delta u}$ to be zero. Figure 5a displays $\rho_{u, \Delta u}$ as a function of $r$ for separation distances up to $L_{u}$ for all three runs. Notice that $\rho_{u, \Delta u}$ is not zero within the inertial subrange for any of the three runs. To gage the importance of these correlations in the inertial subrange, one may compare them to the correlation at $r=L_{u}$, the scale at which the maximum interaction is likely to occur. Notice in Figure $5 \mathrm{a}$, that even after three decades of scale cascading (e.g., Run $\sharp 2$ ), $\rho_{u, \Delta u}$ diminished by one order of magnitude only. Although Runs 1 and 3 did not have such an extensive inertial subrange, the same conclusion holds. Our data were not adequate to test whether $\rho_{u, \Delta u}$ approaches zero for $r \sim \eta$, but it appears to approach that limit gradually for all three runs. This analysis supports the conclusions of Sreenivasan (1991) that anisotropy exists at the small scales despite the existence of an extensive inertial subrange. Using an orthonormal wavelet decomposition of the velocity field, Katul et al. (1994b) also found that localized interactions between large scales and small scales can significantly impact the statistical structure of the inertial subrange in agreement with the above results. If we adopt the argument that this anisotropy is significant, at least for a wide range of scales within the inertial subrange, then we are left with the apparent contradiction as to why (1) and closure assumptions based on local isotropy agree with the measurements in ASL experiments. This question is addressed next.

\subsubsection{Longitudinal Velocity-Thermal Interaction at the Small Scales}

A measure of the net interaction between the small-scale temperature field and the small-scale longitudinal velocity field is given by the correlation coefficient $\rho_{\Delta T, \Delta u}$

$$
\rho_{\Delta T, \Delta u}(r)=\frac{\langle(\Delta T(x, r)-\langle\Delta T(x, r)\rangle)(\Delta u(x, r)-\langle\Delta u(x, r)\rangle)\rangle}{\sigma_{\Delta T} \sigma_{\Delta u}} .
$$



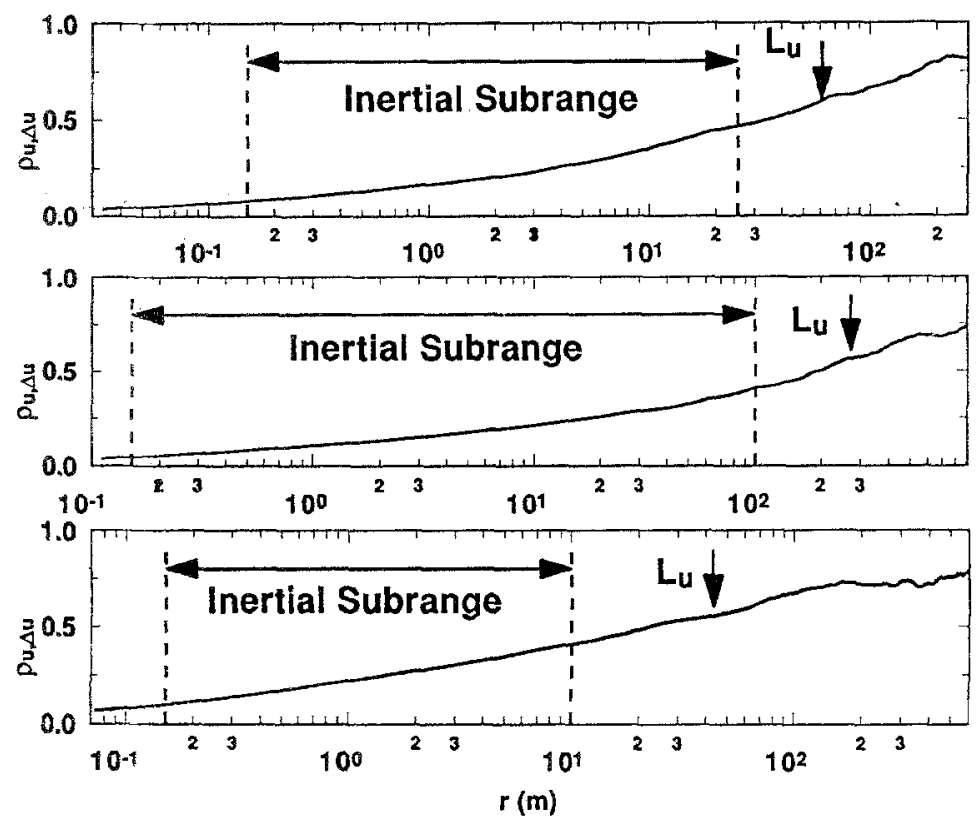

Fig. 5a. The variation of $\rho_{u, \Delta u}$ as a function of $r$ for $r \sim L_{u}$. The inertial subrange is also shown. For locally isotropic turbulence $\rho_{u, \Delta u}=0$. The upper, middle, and lower figures are for Runs 1,2, and 3, respectively.

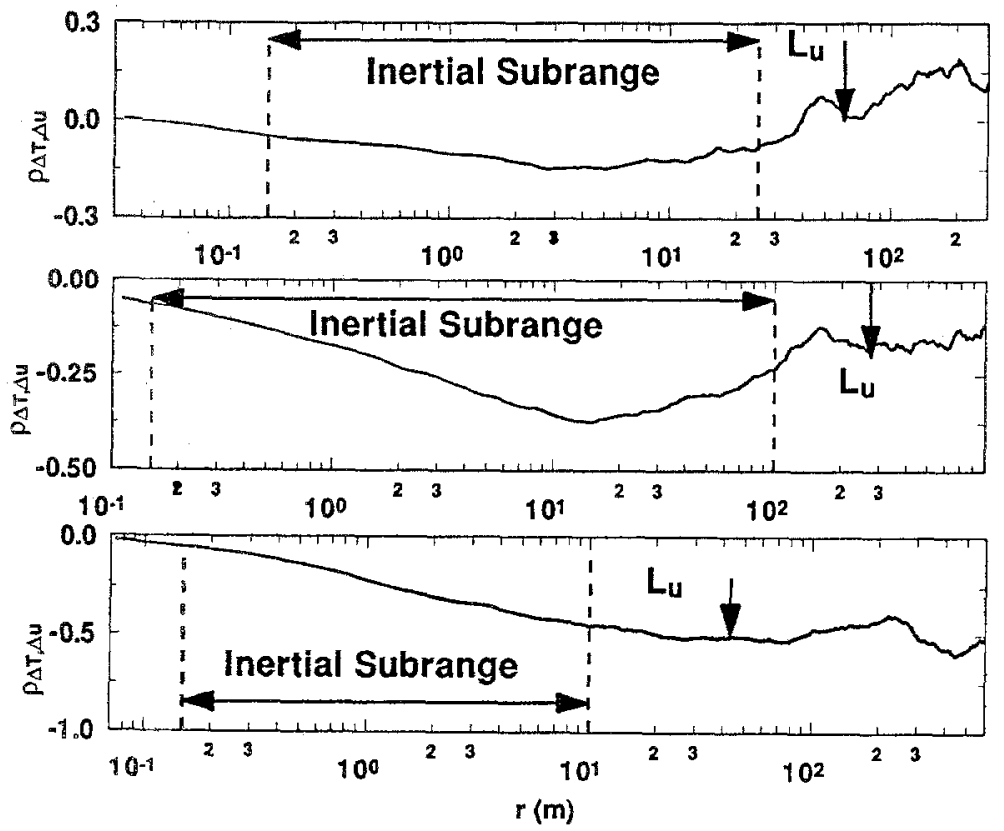

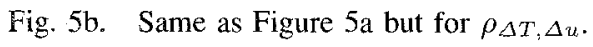



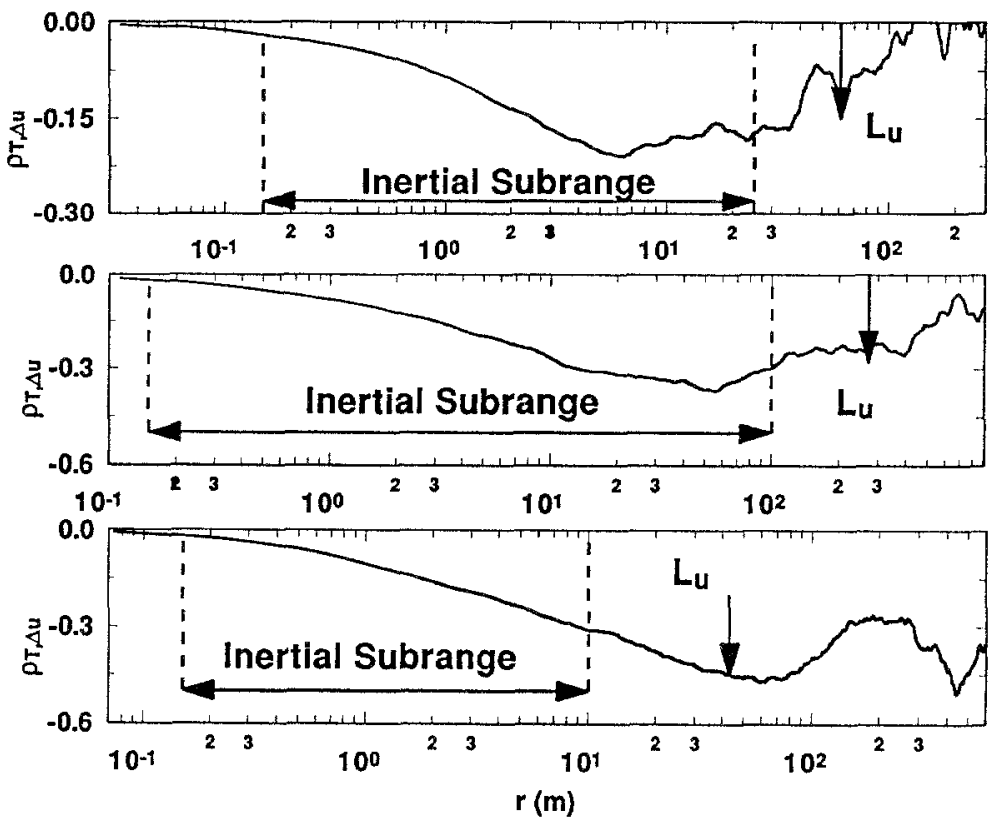

Fig. 5c. Same as Figure 5a but for $\rho_{T, \Delta u}$.

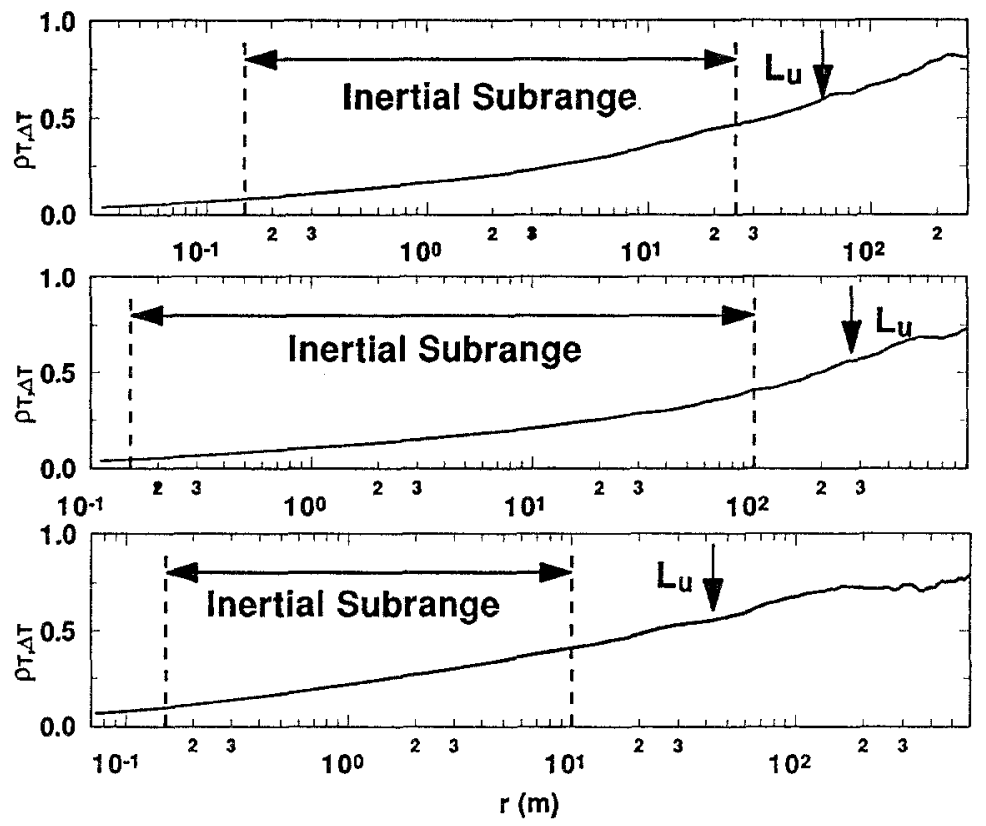

Fig. 5d. Same as Figure 5a but for $\rho_{\Delta T, T}$. 


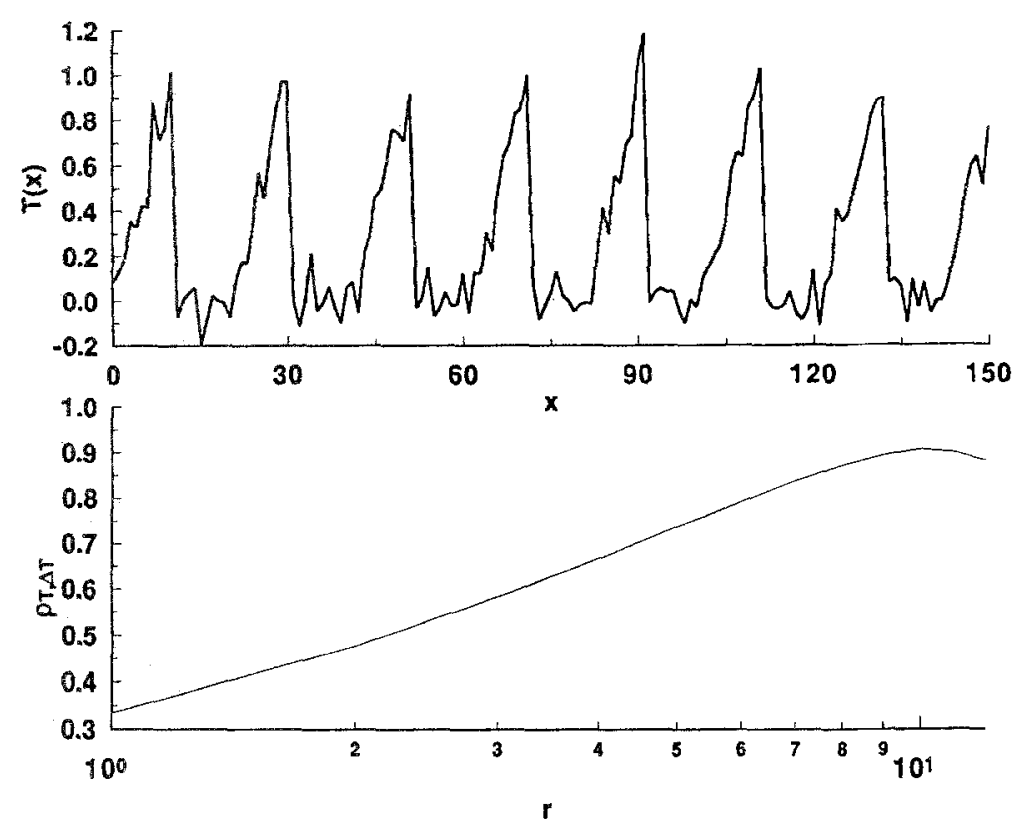

Fig. 5e. The variation of $\rho_{\triangle T, T}$ (lower figure) for an artificial ramp signal (upper figure) perturbed by white noise. The abscissa and ordinate are arbitrary. The ramp size is 10 abscissa units.

The absence of any net interaction requires $\rho_{\Delta T, \Delta u}$ to be zero. As shown by Monin and Yaglom (1975: pp. 99-105), in a locally isotropic turbulence field, $\rho \Delta T, \Delta u$ must vanish. Therefore, we use this correlation to measure local thermal effects on the longitudinal inertial subrange eddy motion. Figure $5 b$ displays $\rho \Delta T, \Delta u$ as a function of $r$ for separation distances up to $L_{u}$ for all three runs. Notice that $\rho_{\Delta T, \Delta u}$ is again not zero within the inertial subrange and is actually comparable in magnitude to $\rho_{u, \Delta u}$ of Figure $5 \mathrm{a}$ for all three runs.

However, what is important to note in Figure $5 b$ is the sign of this correlation. While $\rho_{u, \Delta u}$ is positive, $\rho_{\Delta T, \Delta u}$ is negative, indicating that the anisotropy due to the temperature-velocity interaction counteracts the anisotropy due to largescale/small-scale velocity interactions. This in agreement with the isotropy study reported by Kaimal et al. (1972). In Kaimal et al. (1972), local isotropy was attained very rapidly for strongly unstable conditions, especially when the stability parameter $\left(z / L_{\mathrm{MO}}\right)<-2$. This was not the case for stable conditions (see Figure 22 in Kaimal et al., 1972). Here, $L_{\mathrm{MO}}$ is the Obukhov length given by

$$
L_{\mathrm{MO}}=\frac{-\rho u_{*}^{3}}{k g\left[\frac{H}{c_{p}\langle T\rangle}\right]},
$$

where $u_{*}$ is the friction velocity, $k(=0.4)$ is Von Karman's constant, $g(=9.8 \mathrm{~m}$ $\mathrm{s}^{-2}$ ) is the gravitational acceleration, $z$ is the height above the ground, and $\rho$ is the 
air density. The key difference between the stable case and the strongly unstable case $\left(z / L_{\mathrm{MO}}<-2\right)$ is the relative contributions of buoyant production to the turbulent kinetic energy (TKE). In the case of $\left(z / L_{\mathrm{MO}}\right)<-2$, thermal effects become important ( $H$ is large) and the present analysis reveals that the effect of one anisotropy source is reduced by an opposing anisotropy source (at least along the longitudinal direction). Notice in Kaimal et al. (1972) that when stable conditions prevail (and therefore the sign of the thermal anisotropy reverses), local isotropy is not attained within the range of wavenumbers resolved by their sonic anemometer data. In the stable scenario, both sources of anisotropy have the same sign and produce a superimposed effect (along the longitudinal direction). Similarly, Van Atta (1991) found that buoyancy forces are dynamically important in the inertial subrange for stable stratification. This is a key difference between many neutral laboratory experiments (where $\rho_{\Delta T, \Delta u}=0$ ) and ASL experiments (where $\rho_{\Delta T, \Delta u} \neq 0$ ). Let us illustrate this point further using the Reynolds stress equations with the horizontal homogeneity assumption. These equations are given by

$$
\begin{aligned}
\frac{1}{2} \frac{\partial\left\langle u_{1}^{2}\right\rangle}{\partial t}= & -\left\langle u_{1} u_{3}\right\rangle \frac{\partial\left\langle U_{1}\right\rangle}{\partial z}+\frac{1}{\rho}\left\langle p \frac{\partial u_{1}}{\partial x}\right\rangle-\frac{1}{2} \frac{\partial\left\langle u_{3} u_{1}^{2}\right\rangle}{\partial z}-\nu\left\langle\frac{\partial u_{1}}{\partial x_{j}} \frac{\partial u_{1}}{\partial x_{j}}\right\rangle \\
\frac{1}{2} \frac{\partial\left\langle u_{2}^{2}\right\rangle}{\partial t}= & \frac{1}{\rho}\left\langle p \frac{\partial u_{2}}{\partial y}\right\rangle-\frac{1}{2} \frac{\partial\left\langle u_{3} u_{2}^{2}\right\rangle}{\partial z}-\nu\left\langle\frac{\partial u_{2}}{\partial x_{j}} \frac{\partial u_{2}}{\partial x_{j}}\right\rangle, \\
\frac{1}{2} \frac{\partial\left\langle u_{3}^{2}\right\rangle}{\partial t}= & \frac{g}{T_{a}}\left\langle u_{3} T\right\rangle+\frac{1}{\rho}\left\langle p \frac{\partial u_{3}}{\partial z}\right\rangle-\frac{1}{2} \frac{\partial\left\langle u_{3}^{3}\right\rangle}{\partial z}- \\
& -\nu\left\langle\frac{\partial u_{3}}{\partial x_{j}} \frac{\partial u_{3}}{\partial x_{j}}\right\rangle-\frac{1}{\rho} \frac{\partial\left\langle u_{3} p\right\rangle}{\partial z}
\end{aligned}
$$

where $p$ is the pressure perturbation. The sum of (12), (13), and (14) gives the time evolution of TKE. Notice in (12) and (14) that TKE for unstable ASL flows is produced in two directions, one due to shear, and the other due to buoyancy. Also notice that both temperature and the sensible heat flux $\left\langle u_{3} T\right\rangle$ directly influence the turbulent production in the vertical. Therefore, temperature and sensible heat flux can impact the $u$-component through the pressure-velocity gradient correlations. This component is absent in neutral ASL flows. It is worthwhile here to identify the significance of possible sources of large-scale thermal anisotropy on the local structure of the velocity field.

\subsubsection{Influence of Large-Scale Thermals on the Small-Scale Eddy Motion}

A measure of the net interaction between the large-scale temperature field and the small-scale longitudinal velocity is given by the correlation coefficient $\rho_{T, \Delta u}$

$$
\rho_{T, \Delta u}(r)=\frac{\langle(T(x)-\langle T(x)\rangle)\langle\Delta u(x, r)-\langle\Delta u(x, r)\rangle)\rangle}{\sigma_{T} \sigma_{\Delta u}} .
$$


The absence of any net interaction between the large-scale temperature and small-scale velocity fluctuations requires $\rho_{T, \Delta u}$ to be zero. If this correlation is finite, then the anisotropy characterizing the large-scale thermal motion, namely the ramp-like structure of Figure 3, directly contributes to the small-scale eddy motion of the longitudinal velocity. This can be achieved if one considers the production term in (14). The pressure-redistribution term in (14) is directly influenced by the sensible heat flux and $T$, and this influence might be translated to the small-scale longitudinal eddy motion in (12). The pressure redistribution is thought to diminish this anisotropy and isotropy should prevail within the inertial subrange. Hence, any interaction between $T$ and $\Delta u$ indicates that local isotropy is not achieved. The production source in (14), which is anisotropic, translates to the inertial subrange of (12). Figure $5 \mathrm{c}$ displays $\rho_{T, \Delta u}$ as a function of $r$ for separation distances up to $L_{u}$ for all three runs. Notice that $\rho_{T, \Delta u}$ is not zero within the inertial subrange and is very similar to Figure $5 \mathrm{~b}$ even for the near-neutral case (Run 3).

\subsubsection{Influence of Large-Scale Thermals on the Small-Scale Thermal Motion}

It was pointed out by Sreenivasan et al. (1979) that ramp-like structures (similar to Figure 3) directly contribute to the inertial subrange of the temperature field, and therefore, may directly contribute to inertial subrange anisotropy in the $T_{a}$ measurements. Based on the methodology previously proposed, this interaction should result in significant correlation between $T$ and $\Delta T$. That is,

$$
\rho_{T, \Delta T}(r)=\frac{\langle(T(x)-\langle T(x)\rangle)(\Delta T(x, r)-\langle\Delta T(x, r)\rangle)\rangle}{\sigma_{T} \sigma_{\Delta T}},
$$

should be significant. In Figure 5d, we show $\rho_{\Delta T, T}$ as a function of $r$ for $r$ up to $L_{u}$ and for all three runs. Notice that this correlation is a maximum at sizes comparable to the ramp-like size of Figure $3(\sim 100 \mathrm{~m})$. To explore the hypothesis that this correlation is due to the ramp-like patterns in Figure 3, as suggested by Sreenivasan et al. (1979), let us compare these correlations with those of a signal in which the ramp-like pattern is very well defined.

The influence of ramp-like patterns on the small-scale thermal motion may be better understood if one considers an artificial ramp-like temperature signal, such as the one depicted in Figure 5e, and if one computes $\rho_{\Delta T, T}$ for that signal. In Figure 5e (upper), we show the artificial ramp-like temperature signal perturbed by some white noise and the correlation coefficient $\rho_{\Delta T, T}$ (lower figure) corresponding to that signal. The ramp size of 10 units is approximately uniform throughout the signal. Notice the similarity between the correlations of Figures $5 \mathrm{e}$ and $5 \mathrm{~d}$. This comparison suggests that the finite correlation between $\Delta T$ and $T$ in Figure $5 \mathrm{~d}$ may be due to the ramp-like patterns noted in Figure 3, and therefore, agrees with earlier conclusions by Sreenivasan et al. (1979). Therefore, this analysis suggests that $T$ affects $\Delta T$ through the ramp-like organized structure and $\Delta u$ through the pressure-redistribution term. 


\section{Conclusions}

This study has examined the structure of the inertial subrange from velocity and temperature measurements above a uniform dry lakebed in a desert-like environment. The longitudinal velocity measurements exhibit an inertial subrange that extends about 1.5-3 decades, which is adequate for isotropy investigations. It was found that the Kolmogorov-Obukhov dynamic structure function equation derived from Navier-Stokes for locally isotropic turbulence reproduced the measured second- and third-order structure functions well. Also, Obukhov's constant skewness hypothesis was in good agreement with the measurements. The suggested 0.4 constant skewness value appears to be large. A relation between the constant skewness and Kolmogorov's constant indicated that an $|S|=0.25$ is more reasonable for ASL flows. This 0.25 value also matches our data better. However, these formulations rest on the assumption that the ASL turbulence is locally isotropic. Rather than check the adequacy of local isotropy relations as is commonly performed, we investigated directly the possible sources of anisotropy.

We noted that in a daytime desert-like climate, the sources of anisotropy are due to (1) direct interaction between the large and small scales, and (2) thermal effects on small-scale eddy motion. The second source is absent in many laboratory experiments. In order to explore these two anisotropy sources, we developed statistical measures that are sensitive to such interactions. These statistical measures are simple and very convenient to apply. Using these measures, we found that the large-scale/small-scale interaction was significant in the inertial subrange, thus weakening the local isotropy assumption. It was found that the anisotropy generated by thermal effects was also significant and comparable in magnitude to the large-scale/small-scale interaction anisotropy. However, the thermal anisotropy source was opposite in sign, and thus, counteracted the anisotropy generated by the large-scale/small-scale interaction.

\section{Acknowledgements}

The authors would like to thank Scott Tyler for his assistance and support at Owen's Lake, and Teresa Ortenburger and Mike Mata for their help in data collection. We are grateful for funding support from the National Science Foundation (NSF) grant (EAR-93-04331), United States Geological Survey (USGS), Water Resources Center (WRC) grant (W-812), Kearney Foundation, and UC Davis superfund grant (5 P42ES04699-07).

\section{Appendex 1: Estimation of the Mean Dissipation Rate}

In this study, the mean dissipation rate was used in (1) the Kolmogorov-Obukhov structure function equation, (2) estimating the Taylor microscales, and (3) estimating the Kolmogorov microscale. This appendix discusses how the mean dissipa- 
tion rate was determined. Due to the limited resolution of the sonic anemometer, the dissipation rate was estimated using three distinct methods.

\section{Method 1; production methods}

By estimating the turbulent production rate (mechanical and buoyant) and assuming that the mean production rate is identical to the mean dissipation rate, one can estimate $\langle\epsilon\rangle$ from the similarity relation

$$
\langle\epsilon\rangle=\frac{\phi_{e}\left(z / L_{\mathrm{MO}}\right) u_{*}^{3}}{k z}
$$

where $\phi_{e}($.$) is the stability correction function for the mean dissipation. As shown$ in Panofsky and Dutton (1984: p. 268), the stability correction function $\phi_{e}($.$) is$ given by

$$
\phi_{e}\left(\frac{z}{L_{\mathrm{MO}}}\right)=1-\frac{z}{L_{\mathrm{MO}}} .
$$

The quantity $z / L_{\mathrm{MO}}$ is simply the ratio of the buoyant production $\left(P_{b}\right)$ to the mechanical production $\left(P_{s}\right)$, where $P_{s}$ and $P_{b}$ are given by

$$
P_{s}=\frac{u_{*}^{3}}{k z}, \quad P_{b}=\frac{g H}{\rho c_{p} T_{a}} .
$$

Hence, from the measured $u_{*}, H$, and $T_{a}$, the dissipation rate can be computed. In Table I, we report $P_{s}, P_{b}$, and $P_{s}+P_{b}(=\langle\epsilon\rangle)$. This method is very convenient since no gradient measurements are required. However, the key assumption is the steady-state energy transfer so that the rate of turbulent production is identical to the rate of viscous dissipation.

Method II: local isotropy relations

As shown in Tennekes and Lumley (1972: p. 66), for locally isotropic turbulence, $\langle\epsilon\rangle$ is given by

$$
\langle\epsilon\rangle=15 \nu\left\langle\left(\frac{\partial u_{1}}{\partial x_{1}}\right)^{2}\right\rangle
$$

The space derivative can be computed from the time derivative and Taylor's hypothesis. Cubic spline interpolations are applied prior to the derivative estimation.

Method III: inertial subrange power spectrum

The dissipation rate can also be calculated directly from the power spectrum $E_{u}$ using

$$
E_{u}(K)=C_{1}\langle\epsilon\rangle^{2 / 3} K^{-5 / 3},
$$




\section{TABLE III}

Estimation of the Mean Dissipation Rate using Method III. The regression model $\left(\log \left(E_{u}\right)=A \log (K)+B\right)$ is used. The Coefficient of Determination $\left(R^{2}\right)$, the Standard Error of Estimate (SEE), and the number of data points used $(n)$ are also shown. Deviations from the $-5 / 3$ power law in the slope are used to determine the intermittency exponent $(\mu)$ as discussed in Yakhot et al. (1989)

\begin{tabular}{llllllll}
\hline Run $\sharp$ & $A$ & $B$ & $R^{2}$ & SEE & $n$ & $\langle\epsilon\rangle \times 10^{3}$ & $\mu$ \\
\hline 1 & -1.79 & -1.45 & 0.93 & 0.20 & 4086 & 14.3 & 0.37 \\
2 & -1.79 & -1.93 & 0.94 & 0.19 & 4086 & 3.1 & 0.37 \\
3 & -1.79 & -1.85 & 0.94 & 0.20 & 4086 & 4.1 & 0.37 \\
\hline
\end{tabular}

(see Kaimal and Finnigan, 1994: p. 36; Lumley and Panofsky, 1964: p. 164; McComb, 1990: p. 83; Garrat, 1992: p. 71). This method assumes that K41 is valid with $T_{E}=\langle\epsilon\rangle$. A key disadvantage of this method is the need for a reliable estimate of $C_{1}$.

To determine $\langle\epsilon\rangle$, the velocity power spectrum was first calculated (not shown here). The maximum number of data points that yielded a slope close to $-5 / 3$ were used to estimate the intercept of the regression model $\log E_{u}=A \log$ $K+B$. Here $B=\log \left(C 1\langle\epsilon\rangle^{2 / 3}\right)$. Table III displays the regression statistics used to estimate the dissipation rate of Method III. Notice in Table III that the slopes are larger (in magnitude) than $-5 / 3$. These deviations from the $-5 / 3$ slope are due to intermittency effects intrinsic to the dissipation rate (Kolmogorov, 1962; Monin and Yaglom, 1975; Katul et al., 1994a,b). The estimated intermittency factor $(\mu)$ from runs 1-3 is also shown in Table III. These intermittency factors are in agreement with earlier values $(\mu=0.2-0.5)$ reported in the literature and appear to be independent of stability (see Monin and Yaglom, 1975: Chap. 8; Katul et al., 1994a,b for review).

Methods I and III do not require explicit evaluation of the time derivative. Hence, if the dissipation rate from all methods compares well, the reliability of the estimated Taylor microscale (that is strongly dependent on the accuracy of $\left.\left\langle\left(\partial u_{1} / \partial t\right)^{2}\right\rangle\right)$ can be independently verified. From Table I, the dissipation rates estimated from Methods II and III are in closer agreement when compared to Method I. Despite these differences, all methods predicted the correct magnitude of the dissipation rate. Since Method II provides a direct and explicit estimate of $\langle\epsilon\rangle$ from the NS equations for locally isotropic turbulence, we decided to use the dissipation values from this method. 


\section{Appendix 2: Identification of the Inertial Subrange}

The inertial subrange for all three runs was identified using the third-order structure function given by

$$
D^{3}(r)=\left\langle[u(x+r)-u(x)]^{3}\right\rangle=-\frac{4}{5}\langle\epsilon\rangle r
$$

The following procedure was used in the identification scheme (see e.g., Figure $4 c)$.

(1) Compute $D^{3}(r)$ for $r$ up to $L_{u}$.

(2) Using $\langle\epsilon\rangle$ determined from Method II of Appendix 1 and Table II, plot the line $-4 / 5\langle\epsilon\rangle$.

(3) Identify departures from that line. The upper separation distance at which significant departure is noted marks the upper limit of the inertial subrange.

(4) The lower limit of the inertial subrange was set to $d_{s l}=0.149 \mathrm{~m}$.

Identifying the inertial subrange using the third-order structure function (rather than the usual $-5 / 3$ power law from the power spectrum) has the following advantages:

(a) Intermittency does not affect the identification scheme (see Appendix 1).

(b) The third-order structure function is a higher moment than the power spectrum (second moment) and is therefore more sensitive to small deviations from inertial subrange scaling.

(c) The convergence of $D^{3}(r)$ to $-4 / 5\langle\epsilon\rangle r$ requires a much larger sample size than the convergence of $E_{u}$ to $C_{1}\langle\epsilon\rangle^{2 / 3} \mathrm{~K}^{-5 / 3}$. Also, this convergence is more sensitive to the local isotropy assumption. $\left(D^{3}(r)\right.$ is an odd moment compared to $E_{u}$ which is an even moment.)

The inertial subrange identified from this procedure is presented in Table II for all three runs.

\section{Appendix 3: Relation between the Structure Skewness and Kolmogorov's Constant}

As discussed earlier, the uncertainty in the structure skewness is very large (0.20.5 ) with an 0.4 suggested value based on grid turbulence measurements. It is therefore convenient to relate $|S|$ to some well known constant in the ASL. The structure skewness $S\left(=D^{3}(r)\left[D^{2}(r)\right]^{-3 / 2}\right)$ can be related to Kolmogorov's constant $C_{1}$ (see Appendix 1) for a locally isotropic turbulence if

(1) $\mathrm{K} 41$ is valid so that $D^{2}(r)=C_{s}\langle\epsilon\rangle^{2 / 3} r^{2 / 3}$, where $C_{s}\left(=4 C_{1}\right)$ is the Kolmogorov constant based on the second-order structure function definition (see Kaimal and Finnigan, 1994: p. 64).

(2) $D^{3}(r)=-4 / 5\langle\epsilon\rangle r$.

(3) Intermittency corrections $(\mu)$ to K41 (at second order) are negligible. 
Hence, with $D^{3}(r)$ known from the asymptotic result of the KolmogorovObukhov structure function equation, and with $D^{2}(r)$ related to the Kolmogorov constant from K41, $S$ can be related to $C_{1}$ by

$$
S=\frac{D^{3}(r)}{\left[D^{2}(r)\right]^{3 / 2}}=-\frac{4}{5}\left(4 C_{1}\right)^{-3 / 2} \simeq 0.1 C_{1}^{-3 / 2}
$$

Hence, for $C_{1}=0.55$ (see Kaimal and Finnigan, 1994), $|S|=0.25$, which is less than the accepted 0.4 value.

\section{References}

Antonia, R. A. and Chambers, A. J.: 1980, 'On the Correlation between Turbulent Velocity and Temperature Derivatives in the Atmospheric Surface Layer', Boundary-Layer Meteorol. 18, $399-410$.

Antonia, R. A. and Van Atta, C. W.: 1975, 'On the Correlation between Temperature and Velocity Dissipation Fields in a Heated Turbulent Jet', J. Fluid Mech. 67, 273-288.

Antonia, R. A. and Raupach, M. R.: 1993, 'Spectral Scaling in a High Reynolds Number Laboratory Boundary Layer', Boundary-Layer Meteorol. 65, 289-306.

Bradley, E. F., Antonia, R. A., and Chambers, A.J.: 1981, 'Turbulence Reynolds Number and the Turbulent Kinetic Energy Balance in the Atmospheric Surface Layer', Boundary-Layer Meteorol. 21, 183-197.

Frisch, U., Sulem, P., and Nelkin, M.: 1978, 'A Simple Dynamical Model of Intermittent Fully Developed Turbulence', J. Fluid Mech. 87, 719-736.

Friehe, C. A.: 1986, 'Fine-Scale Measurements of Velocity, Temperature, and Humidity in Atmospheric Surface Layer', in Lenschow, D. (ed.), Probing the Atmospheric Boundary Layer, American Meteorological Society, $269 \mathrm{pp}$.

Garratt, J. R.: 1992, The Atmospheric Boundary Layer, Cambridge University Press, 316 pp.

Kaimal, J. C., Wyngaard, J. C., Izumi, Y., Coté, O. R.: 1972, 'Spectral Characteristics of Surface Layer Turbulence', Quart. J. R. Meteorol. Soc. 98, 563-589.

Kaimal, J. C. and Finnigan, J. J.: 1994, Atmospheric Boundary Layer Flows: Their Structure and Measurements, Oxford University Press, $289 \mathrm{pp}$.

Katul, G. G., Parlange, M. B., and Chu, C. R.: 1994a, 'Intermittency, Local Isotropy, and nonGaussian Statistics in Atmospheric Surface Layer Turbulence', Physics of Fluids, 6, 2480-2492.

Katul, G. G.: 1994, 'A Model for Sensible Heat Flux Probability Density Function for Near-Neutral and Slightly-Stable Atmospheric Flows', Boundary-Layer Meteorol., in press.

Katul, G. G., Albertson, J. D., Chu, C. R., and Parlange, M. B.: 1994b, 'Intermittency in Atmospheric Surface Layer Turbulence: The Orthonormal Wavelet Representation', in E. FoufoulaGeorgiou and P. Kumar (eds.), Wavelets in Geophysics, Academic Press, 365 pp.

Kerr, R. M.: 1990, 'Velocity, Scalar, and Transfer Spectra in Numerical Turbulence', J. Fluid Mech. 211, 309-332.

Kim, J. and Antonia, R. A.: 1993, 'Isotropy of the Small Scales of Turbulence at Low Reynolds Number', J. Fluid Mech. 251, 219-238.

Kolmogorov, A. N.: 1941, 'The Local Structure of Turbulence in Incompressible Viscous Fluid for Very Large Reynolds Number', Dokl. Akad. Nauk. SSSR 30, 301-303.

Kolmogorov, A. N.: 1962, 'A Refinement of Previous Hypothesis Concerning the Local Structure of Turbulence in a Viscous Incompressible Fluid at High Reynolds Number', J. Fluid Mech. $13,82-85$.

Landau, L. D. and Lifshitz, E. M.: 1986, Fluid Mechanics, Pergamon Press, 539 pp.

Lesieur, M.: 1987, Turbulence in Fluids, Martinus Nijhoff Publishers, 286 pp.

Lumley, J. and Panofsky, H.: 1964, The Structure of Atmospheric Turbulence, John Wiley and Sons, 229 pp. 
Lumley, J.: 1965, 'Interpretation of Time Spectra Measured in High Intensity Shear Flows', Phys. Fluids 6, 1056-1062.

Mahrt, L.: 1989, 'Intermittency of Atmospheric Turbulence', J. Atmos. Sci. 46, 79-95.

McComb, W. D.: 1990, The Physics of Fluid Turbulence, Oxford Science Publications, 572 pp.

Mestayer, P.: 1982, 'Local Isotropy and Anisotropy in a High Reynolds Number Turbulent Boundary Layer', J. Fluid Mech. 125, 475-503.

Monin, A. S. and Yaglom, A. M.: 1975, Statistical Fluid Mechanics, MIT Press, 875 pp.

Obukhov, A. M.: 1949, 'Local Structure of Atmospheric Turbulence', Dokl. Akad. Nauk. SSSR, 67, 643-646.

Panofsky, H. and Dutton, J.: 1984, Atmospheric Turbulence: Models and Methods for Engineering Applications, John Wiley and Sons, $397 \mathrm{pp}$.

Praskovsky, A. A.: 1992, 'Experimental Verification of the Kolmogorov Refined Similarity Hypothesis', Phys. Fluids A 4, 2589-2591.

Praskovsky, A. A., Gledzer, E. B., Karyakin, M. Y., and Zhou, Y.: 1993, 'The Sweeping Decorrelation Hypothesis and Energy-Inertial Scale Interaction in High Reynolds Number Flows', $J$. Fluid Mech. 248, 493-511.

Sirivat, A. and Warhaft, Z,: 1983, 'The Effect of a Passive Cross-Stream Temperature Gradient on the Evolution of Temperature Variance and Heat Flux in Grid Turbulence', J. Fluid Mech. 128, 323-346.

Suomi, V. E. and Businger, J. A.: 1959, 'Sonic Anemometer-Thermometer', Geophys. Res. Papers $59,1-13$.

Sreenivasan, K. R., Antonia, R. A., and Britz, D.: 1979, 'Local Isotropy and Large Structures in a Heated Turbulent Jet', J. Fluid Mech. 94, 745-775.

Sreenivasan, K. R.: 1991, 'On Local Isotropy of Passive Scalars in Turbulent Shear Flows', in J. C. R. Hunt, O. M. Phillips, and D. Williams (eds.), Turbulence and Stochastic Processes: Kolmogorov's Ideas 50 Years On, Roy. Soc., 240 pp.

Stull, R.: 1988, An Introduction to Boundary Layer Meteorology, Kluwer Academic Press, 666 pp. Taylor, G. I.: 1938, 'The Spectrum of Turbulence', Proc. Roy. Soc., A CLXIV, 476-490.

Tennekes, H. and Lumley, J. L.: 1972, A First Course in Turbulence, MIT Press, 300 pp.

Townsend, A. A.: 1976, The Structure of Turbulent Shear Flow, Cambridge University Press, 429 pp.

Van Atta, C. W. and Chen, W. Y.: 1970, 'Structure Functions of Turbulence in the Atmospheric Boundary Layer over the Ocean', J. Fluid Mech. 44, 145-159.

Van Atta, C.: 1991, 'Local Isotropy of the Smallest Scales of Turbulent Scalar and Velocity Fields', in J. C. R. Hunt, O. M. Phillips, and D. Williams (eds.), Turbulence and Stochastic Processes: Kolmogorov's Ideas 50 Years On, Roy. Soc., $240 \mathrm{pp}$.

Wyngaard, J. C.: 1981, 'Cup, Propeller, Vane, and Sonic Anemometer in Turbulence Research', Ann. Rev. Fluid Mech. 13, 922-929.

Yakhot, V., She, Z. S., and Orzag, S. A.: 1989, 'Deviations from the Classical Kolmogorov Theory of the Inertial Subrange of Homogeneous Turbulence', Phys. Fluids 2, 289-293.

Yamada, M. and Ohkitani, K.: 1991, 'Orthonormal Wavelet Analysis of Turbulence', Fluid Dynamics Res. 8, 101-115. 